T. NODA

KODAI MATH. J.

22 (1999), 66-82

\title{
ON THE ZEROS OF SYMMETRIC SQUARE $L$-FUNCTIONS
}

\author{
TAKUMI NODA
}

\section{Introduction}

Let $k$ be a positive even integer and $S_{k}$ be the space of cusp forms of weight $k$ on $S L_{2}(Z)$. Let $f(z) \in S_{k}$ be a normalized Hecke eigenform with the Fourier expansion $f(z)=\sum_{n=1}^{\infty} a(n) e^{2 \pi i n z}$. The symmetric square $L$-function attached to $f(z)$ is defined by

$$
L_{2}(s, f)=\prod_{p}\left(1-\alpha_{p}^{2} p^{-s}\right)^{-1}\left(1-\alpha_{p} \beta_{p} p^{-s}\right)^{-1}\left(1-\beta_{p}^{2} p^{-s}\right)^{-1},
$$

with $\alpha_{p}+\beta_{p}=a(p)$ and $\alpha_{p} \beta_{p}=p^{k-1}$. Here the product is taken over all rational primes. We denote $L(s, f, \underline{s t}):=L_{2}(s+k-1, f)$ and call $L(s, f, \underline{s t})$ the standard $L$-function attached to $f(z)$ on $S L_{2}(Z)$.

In an application of the projection operator on the space of $C^{\infty}$-automorphic forms introduced by Sturm [St] and the holomorphy of $L(s, f, s t)$ by Shimura [Sh 1], we gave one formula which connects the zeros of zeta-functions with the eigenvalues of the Hecke operators on $S L_{2}(Z)$ (cf. [Nd]); and we saw the distributions of the zeros of the Riemann zeta-function and of symmetric square $L$-functions are explicitly connected with the distribution of the Hecke eigenvalues. Therefore it is natural to ask the relation of the zeros of automorphic $L$ functions to the Fourier coefficients of automorphic forms when the $L$-function has a "good" integral representation.

In this paper we consider the zeros of symmetric square $L$-functions again. Regarding the symmetric square $L$-functions as the standard $L$-functions in the sense of [An], we are able to apply some results of Siegel modular forms by [Bö, Mi]; and we shall derive one formula as Theorem 1 which connects the zeros of $L(s, f, \underline{s t})$ with the Fourier coefficients of non-holomorphic Eisenstein series for Siegel modular group $\operatorname{Sp}(2, Z)$.

\section{Statements of results}

For $n \in Z_{>0}$, let $\Gamma^{n}:=S p(n, Z)$ be the Siegel modular group of degree $n$ and let $H_{n}$ be the Siegel upper half space of degree $n$. If $a$ is an $m \times n$-matrix, then we write it also as $a^{(m, n)}$, and as $a^{(m)}$ if $m=n$. We denote by ${ }^{t} a$ the transpose of $a$, and by $a_{i j}$ the $(i, j)$-entry of $a$. For two matrices $a$ and $b$, we put $a[b]:={ }^{t} b a b$

Recelved May 12, 1998. 
if the right-hand side is defined. The identity matrix (resp. zero matrix) of size $m$ is denoted by $1_{m}\left(\right.$ resp. $\left.0_{m}\right)$. For a commutative ring $R$, we denote by $R^{(m, n)}$ the $R$-module of all $m \times n$-matrices with entries in $R$. We put $R^{(m)}:=R^{(m, n)}$,

$$
\begin{aligned}
V_{m} & :=\left\{\left.x \in \boldsymbol{R}^{(m)}\right|^{t} x=x\right\}, \\
P_{m} & :=\left\{x \in V_{m} \mid x>0\right\}, \\
\kappa(v) & :=\frac{v+1}{2} \quad \text { for } v \in Z_{\geqq 0} .
\end{aligned}
$$

For square matrices $a_{1}, \ldots, a_{n}$, we write

$$
\operatorname{diag}\left(a_{1}, \ldots, a_{n}\right)=\left(\begin{array}{ccc}
a_{1} & & 0 \\
& \ddots & \\
0 & & a_{n}
\end{array}\right) .
$$

The set of symmetric half-integral matrices of size $m$ is denoted by $\Lambda_{m}$, and we put

$$
\Lambda_{m}^{*}:=\left\{h \in \Lambda_{m} \mid \operatorname{det}(h) \neq 0\right\} .
$$

Let $\sigma$ the trace for matrices and $e(x):=\exp (2 \pi i x)$. For $h \in \Lambda_{v}$ and $s \in C$, the singular series (Siegel series) is defined by

$$
S_{v}(h, s):=\sum_{r \in V_{v} \cap Q^{(v)} \bmod 1} n(r)^{-s} \boldsymbol{e}(\sigma(h r)),
$$

where $n(r)$ is the product of reduced positive denominators of the elementary divisors of $r$. Especially

$$
S_{1}(h, s)=\sum_{k=1}^{\infty} \sum_{\substack{l=1 \\(l, k)=1}}^{k-1} \exp \left(2 \pi i h \times \frac{l}{k}\right)=d_{1-s}(h) \cdot \zeta(s)^{-1}
$$

and by [Bö 1, Ki] (cf. [Mi, (5.1)]),

$$
S_{2}\left(\left(\begin{array}{ll}
h & 0 \\
0 & 0
\end{array}\right), s\right)=\frac{\zeta(2 s-3) \cdot d_{2-s}(h)}{\zeta(2 s-2) \cdot \zeta(s)} .
$$

Here $d_{s}(h)$ is the sum of the $s$-th powers of positive divisors of $h$. For $m>0$, we put

$$
\Gamma_{m}(s):=\pi^{m(m-1) / 4} \prod_{j=0}^{m-1} \Gamma\left(s-\frac{j}{2}\right)
$$

and $\Gamma_{0}(s):=1$. For $g \in P_{m}, h \in V_{m}$, and $(\alpha, \beta) \in C^{2}$, we put

$$
\eta_{m}(g, h ; \alpha, \beta):=\int_{\substack{x \pm h>0 \\ V_{m}}} e^{-\sigma(g x)} \operatorname{det}(x+h)^{\alpha-\kappa(m)} \operatorname{det}(x-h)^{\beta-\kappa(m)} d x
$$


with $d x=\prod_{l \leqq j} d x_{i j}$, which is convergent for $\operatorname{Re}(\alpha)>\kappa(m)-1$ and $\operatorname{Re}(\beta)>m$. Concerning confluent hypergeometric function, we also use the following ones defined in [Sh 2]: For $g \in P_{m}, h \in V_{m}$ and $(\alpha, \beta) \in C^{2}$,

$$
\xi_{m}(g, h ; \alpha, \beta):=\int_{V_{m}} \boldsymbol{e}(-\sigma(h x)) \operatorname{det}(x+i g)^{-\alpha} \operatorname{det}(x-i g)^{-\beta} d x
$$

which satisfies

$$
\xi_{m}(g, h ; \alpha, \beta):=e^{(\pi / 2) i m(\beta-\alpha)} 2^{m} \pi^{m \kappa(m)} \Gamma_{m}(\alpha)^{-1} \Gamma_{m}(\beta)^{-1} \eta_{m}(2 g, \pi h ; \alpha, \beta) .
$$

Our main purpose is to prove the following theorem:

THEOREM 1. Let $\Delta_{k}(z) \in S_{k}$ be the unique normalized Hecke eigenform for $k=12,16,18,20,22$, and 26 . Let $\rho$ be a zero of $L\left(s, \Delta_{k}, \underline{s t}\right)$ in the critical strip $0<\operatorname{Re}(s)<1$, with $\rho \notin R$ and $\zeta(2 \rho) \neq 0$. Then for each positive integer $n$ and for any $t>0$,

$$
\begin{aligned}
& \sum_{\substack{h, p, q \in Z_{>0} \\
h q^{2}=n(p, q)=1}}(-1)^{(k / 2)+1} 2^{-\bar{\rho}} \pi \cdot \Gamma\left(\frac{\bar{\rho}+k-1}{2}\right) \Gamma\left(\frac{\bar{\rho}+k+1}{2}\right)^{-1} d_{-\bar{\rho}}(h) p^{-\bar{\rho}+k-1} q^{-\bar{\rho}-k+1} e^{-2 \pi t h p^{2}} \\
& =\int_{0}^{\infty} \sum_{h \in \Lambda_{2}^{*} h=\left(\begin{array}{c}
* \\
* \\
n
\end{array}\right)} \zeta(\bar{\rho}+1) S_{2}(h, \bar{\rho}+1) y^{(\bar{\rho}+k-3) / 2} e^{-2 \pi n y} \\
& \quad \times \xi_{2}\left(\left(\begin{array}{ll}
t & 0 \\
0 & y
\end{array}\right), h, \frac{\bar{\rho}+k+1}{2}, \frac{\bar{\rho}-k+1}{2}\right) d y .
\end{aligned}
$$

\section{3. $C^{\infty}$-Siegel modular forms}

For $k \in Z_{\geqq 0}$, we denote by $S_{k}^{n}$ the set of cusp forms of weight $k$ on $\operatorname{Sp}(n, Z)$ and by $\mathfrak{M}_{k}^{n}$ the set of functions $F$ which satisfy the following conditions:

(3-A) $F$ is a $C^{\infty}$ function from $H_{n}$ to $C$,

(3-B) $\quad F\left((a z+b)(c z+d)^{-1}\right)=\operatorname{det}(c z+d)^{k} F(z) \quad$ for all $\left(\begin{array}{ll}a^{(n)} & b^{(n)} \\ c^{(n)} & d^{(n)}\end{array}\right) \in \Gamma^{n}$.

The function $F$ is called a $C^{\infty}$-modular form on $\Gamma^{n}$ of weight $k$, and called of bounded growth if for every $\varepsilon>0$

where

$$
\int_{X} \int_{Y}|F(z)| \operatorname{det}(y)^{k-1-n} e^{-\varepsilon \sigma(y)} d y d x<\infty,
$$

$$
X=\left\{x \mid x \in V_{n}(\bmod 1)\right\} \quad \text { and } \quad Y=\left\{y \mid y \in P_{n}\right\} .
$$

In this paper we denote $d x=\prod_{l \leqq J} d x_{i j}$ and $d y=\prod_{l \leqq J} d y_{i j}$. For $F \in \mathfrak{M}_{k}^{n}$ and 
$f \in S_{k}^{n}$, the Petersson inner product is defined by

$$
\langle F, f\rangle=\int_{\Gamma^{n} \backslash H_{n}} F(z) \overline{f(z)} \operatorname{det}(y)^{k-n-1} d x d y .
$$

Here $z=x+i y$ with real matrices $x$ and $y$; we also denote $\operatorname{Re}(z)=x$ and $\operatorname{Im}(z)=y$.

We shall quote the following theorem:

TheOREM A ([St], Theorem 1). Let $F \in \mathfrak{M}_{k}^{n}$ be of bounded growth with the Fourier expansion $F(z)=\sum_{T \in \Lambda_{n}} a(T, y) e^{2 \pi i \sigma(T x)}$. Assume $k>2 n$. Let

$$
\begin{aligned}
c(T)= & \Gamma_{n}\left(k-\frac{(n-1)}{2}\right)^{-1} \pi^{n(k-(n+1) / 2)} \operatorname{det}(4 T)^{(k-(n+1) / 2)} \\
& \times \int_{Y} a(T, y) e^{-2 \pi \sigma(T y)} \operatorname{det}(y)^{k-1-n} d y .
\end{aligned}
$$

Then $h(z)=\sum_{T \in \Lambda_{n} T>0} c(T) e^{2 \pi \imath \sigma(T z)} \in S_{k}^{n}$ and $\langle g, F\rangle=\langle g, h\rangle$ for all $g \in S_{k}^{n}$.

We shall also use the following properties of the function $L(s, f, \underline{s t})$. We put

$$
\Lambda(s, f, \underline{s t}):=\Gamma_{\boldsymbol{R}}(s+\varepsilon) \prod_{j=1}^{n} \Gamma_{C}(s+k-j) L(s, f, \underline{s t})
$$

with

$$
\Gamma_{\boldsymbol{R}}(s):=\pi^{-s / 2} \Gamma\left(\frac{s}{2}\right), \quad \Gamma_{\boldsymbol{C}}(s):=2(2 \pi)^{-s} \Gamma(s)
$$

and

$$
\varepsilon:= \begin{cases}0 & \text { for } n \text { even } \\ 1 & \text { for } n \text { odd }\end{cases}
$$

Let $k$ be an even integer, then the function $\Lambda(s, f, \underline{s t})$ has the following integral representation ([Bö 2], Satz 5):

$$
\Lambda(s, f, \underline{s t}) \cdot f(z)=\alpha_{k}^{(n)}(s) \zeta(s+n) \prod_{j=1}^{n-1} \zeta(2 s+2 j)\left\langle f, E_{k}^{(2 n)}\left(\left(\begin{array}{cc}
-\bar{z} & 0 \\
0 & *
\end{array}\right), \frac{\bar{s}-k+1}{2}\right)\right\rangle
$$

with

$$
\alpha_{k}^{(n)}(s):=(-1)^{n k / 2} 2^{\left(n^{2}-3 n\right) / 2-1+n s} \pi^{-n((n / 2)+s+k)-(1 / 2)(s+k)} \Gamma\left(\frac{s+\varepsilon}{2}\right) \Gamma_{n}\left(\frac{s+k}{2}\right) \Gamma_{n}\left(\frac{s+k+n}{2}\right) .
$$

Here $E_{k}^{(n)}(z, s)$ is the Eisenstein series

$$
E_{k}^{(n)}(z, s)=\operatorname{det}(\operatorname{Im}(z))^{s} \sum_{\{c, d\}} \operatorname{det}(c z+d)^{-k}|\operatorname{det}(c z+d)|^{-2 s}
$$


for $n \in Z_{>0}$ and $z \in H_{n}$. The summation is taken over $\left(\begin{array}{cc}* & * \\ c^{(n)} & d^{(n)}\end{array}\right)$, a complete system of representation of $\left\{\left(\begin{array}{cc}* & * \\ 0^{(n)} & *\end{array}\right) \in \Gamma^{n}\right\} \backslash \Gamma^{n}$.

\section{Eisenstein series}

The purpose of this section is to prepare some properties of the Eisenstein series. For Siegel modular group, the explicit Fourier expansion and growth of the Eisenstein series were studied by Mizumoto [Mi]. We shall write down the expansion in the case of degree 2 and show the Eisenstein series is of bounded growth in our case.

First, we introduce some notations. For $v \in Z$ with $1 \leqq v \leqq m$,

$$
Z_{p r m}^{(m, v)}:=\left\{a \in \boldsymbol{Z}^{(m, v)} \mid a \text { is primitive }\right\} .
$$

For $1 \leqq v \leqq m$ and $y \in P_{m}$, let

$$
\zeta_{v}^{(m)}(g, s):=\sum_{a \in \boldsymbol{Z}_{p r m}^{(m, v)} / G L_{v}(\boldsymbol{Z})} \operatorname{det}(g[a])^{-s} .
$$

The right-hand side of (4.1) converges locally uniformly for $\operatorname{Re}(s)>m / 2$. By [Ma], the function $\zeta_{v}^{(m)}(g, s)$ has a meromorphic continuation to the whole $s$ plane. By definition,

$$
\zeta_{m}^{(m)}(g, s)=\operatorname{det}(g)^{-s}
$$

We also put $\zeta_{0}^{(m)}(*, s):=1$ for all $m \in Z_{\geqq 0}$.

We also use

$$
\eta_{m}^{*}(g, h ; \alpha, \beta):=\operatorname{det}(g)^{\alpha+\beta-\kappa(m)} \eta_{m}(g, h ; \alpha, \beta) .
$$

Here $\eta_{m}(g, h ; \alpha, \beta)$ is defined by $(2.2)$.

For $1 \leqq \lambda \leqq m$ and $y \in P_{m}$, we use the notation as defined in [Mi]:

$$
y\left[u_{r}\right]=\operatorname{diag}\left(y[r], g\left(y, u_{r}\right)\right)\left[\begin{array}{cc}
1_{\lambda} & b \\
0 & 1_{m-\lambda}
\end{array}\right]
$$

or

$$
g\left(y, u_{r}\right):=y\left[r_{1}\right]-(y[r])^{-1}\left[{ }^{t} r y r_{1}\right]
$$

if $u_{r}=\left(r r_{1}\right) \in G L_{m}(\boldsymbol{Z})$ and $r \in \boldsymbol{Z}_{p r m}^{(m, v)}$.

Now we quote the Fourier expansion of Eisenstein series:

THEOREM B ([Mi], Theorem 1.8). (1) For $m \in Z_{>0}, k \in 2 Z_{\geqq 0}$, and $\operatorname{Re}(s)>$ $m$, the Eisenstein series $E_{k}^{(m)}(z, s)$ has the following expression:

$$
E_{k}^{(m)}(z, s)=\sum_{v=0}^{m} \sum_{\lambda=0}^{v} F_{k, v, \lambda}^{(m)}(z, s)
$$


where

$$
\begin{aligned}
F_{k, v, 0}^{(m)}(z, s)= & (-1)^{k v / 2} 2^{v} \pi^{v k(v)} \frac{\Gamma_{v}(2 s+k-\kappa(v))}{\Gamma_{v}(s) \Gamma_{v}(s+k)} \\
& \cdot S_{v}\left(0_{v}, 2 s+k\right) \operatorname{det}(y)^{s} \zeta_{v}^{(m)}(2 y, 2 s+k-\kappa(v))
\end{aligned}
$$

for $0 \leqq v \leqq m$, and

$$
F_{k, v, \lambda}^{(m)}(z, s)=\sum_{h \in \Lambda_{\lambda}^{*}} \sum_{r \in \boldsymbol{Z}_{p r i m}^{(m, \lambda)} / G L_{\lambda}(\boldsymbol{Z})} b_{k, v, \lambda}^{(m)}\left(h\left[{ }^{t} r\right], y, s\right) \boldsymbol{e}\left(\sigma\left(h\left[{ }^{t} r\right] x\right)\right)
$$

for $1 \leqq \lambda \leqq v \leqq m$ with

$$
\begin{aligned}
\left.b_{k, v, \lambda}^{(m)}\left(h{ }^{t} r\right], y, s\right):= & (-1)^{k v / 2} 2^{v} \pi^{v \kappa(v)+\lambda((v-\lambda) / 2)} \frac{\Gamma_{v-\lambda}(2 s+k-\kappa(v))}{\Gamma_{v}(s) \Gamma_{v}(s+k)} \\
& \cdot S_{v}\left(\operatorname{diag}\left(h, 0_{v-\lambda}\right), 2 s+k\right) \operatorname{det}(y)^{s} \operatorname{det}(2 y[r])^{\kappa(v)-k-2 s} \\
& \cdot \eta_{\lambda}^{*}\left(2 y[r], \pi h ; s+k+\frac{\lambda-v}{2}, s+\frac{\lambda-v}{2}\right) \\
& \cdot \zeta_{v-\lambda}^{(m-\lambda)}\left(2 g\left(y, u_{r}\right), 2 s+k-\kappa(v)\right) .
\end{aligned}
$$

(2) Above (4.2)-(4.5) give the analytic continuation of $E_{k}^{(m)}(z, s)$ to the whole s-plane. Moreover $E_{k}^{(m)}(z, s)$ is termwise differentiable up to any order in the entries of $x$ and $y$ except on the poles of $E_{k}^{(m)}(z, s)$.

We use the Theorem $\mathbf{B}$ in the case of $m=2$ :

$$
E_{k}^{(2)}\left(\left(\begin{array}{cc}
-\bar{z} & 0 \\
0 & w
\end{array}\right), \frac{\bar{s}-k+1}{2}\right)=\sum_{v=0}^{2} \sum_{\lambda=0}^{v} F_{k, v, \lambda}^{(2)}\left(\left(\begin{array}{cc}
-\bar{z} & 0 \\
0 & w
\end{array}\right), \frac{\bar{s}-k+1}{2}\right),
$$

where

$$
\begin{aligned}
F_{k, 0,0}^{(2)}\left(\left(\begin{array}{cc}
-\bar{z} & 0 \\
0 & w
\end{array}\right), \frac{\bar{s}-k+1}{2}\right)=\operatorname{det}\left(\operatorname{Im}\left(\begin{array}{cc}
z & 0 \\
0 & w
\end{array}\right)\right)^{(\bar{s}-k+1) / 2}, \\
F_{k, 1,0}^{(2)}\left(\left(\begin{array}{cc}
-\bar{z} & 0 \\
0 & w
\end{array}\right), \frac{\bar{s}-k+1}{2}\right) \\
=(-1)^{k / 2} 2 \pi \frac{\Gamma(\bar{s})}{\Gamma((\bar{s}-k+1) / 2) \Gamma((\bar{s}+k+1) / 2)} \\
\cdot S_{1}\left(0_{1}, \bar{s}+1\right) \operatorname{det}\left(\operatorname{Im}\left(\begin{array}{cc}
z & 0 \\
0 & w
\end{array}\right)\right)^{(\bar{s}-k+1) / 2} \zeta_{1}^{(2)}\left(2 \operatorname{Im}\left(\begin{array}{cc}
z & 0 \\
0 & w
\end{array}\right), \bar{s}\right)
\end{aligned}
$$




$$
\begin{aligned}
= & (-1)^{k / 2} 2 \pi \frac{\Gamma(\bar{s})}{\Gamma((\bar{s}-k+1) / 2) \Gamma((\bar{s}+k+1) / 2)} \\
& \cdot \zeta(\bar{s}) \zeta(\bar{s}+1)^{-1} \operatorname{det}\left(\operatorname{Im}\left(\begin{array}{cc}
z & 0 \\
0 & w
\end{array}\right)\right)^{(\bar{s}-k+1) / 2} \zeta_{1}^{(2)}\left(2 \operatorname{Im}\left(\begin{array}{cc}
z & 0 \\
0 & w
\end{array}\right), \bar{s}\right),
\end{aligned}
$$

$$
\begin{aligned}
F_{k, 2,0}^{(2)}( & \left.\left(\begin{array}{cc}
-\bar{z} & 0 \\
0 & w
\end{array}\right), \frac{\bar{s}-k+1}{2}\right) \\
= & (-1)^{k / 2} 2^{2} \pi^{2} \frac{\Gamma(\bar{s}-(1 / 2)) \Gamma(\bar{s}-1)}{\Gamma((\bar{s}-k+1) / 2) \Gamma((\bar{s}-k) / 2) \Gamma((\bar{s}+k+1) / 2) \Gamma((\bar{s}+k) / 2)} \\
& \cdot S_{2}\left(0_{2}, \bar{s}+1\right) \operatorname{det}\left(\operatorname{Im}\left(\begin{array}{cc}
z & 0 \\
0 & w
\end{array}\right)\right)^{(\bar{s}-k+1) / 2} \zeta_{2}^{(2)}\left(2 \operatorname{Im}\left(\begin{array}{cc}
z & 0 \\
0 & w
\end{array}\right), \bar{s}-\frac{1}{2}\right) \\
= & (-1)^{k / 2} 2^{2} \pi^{2} \frac{\Gamma(\bar{s}-(1 / 2)) \Gamma(\bar{s}-1)}{\Gamma((\bar{s}-k+1) / 2) \Gamma((\bar{s}-k) / 2) \Gamma((\bar{s}+k+1) / 2) \Gamma((\bar{s}+k) / 2)} \\
& \cdot \frac{\zeta(2 \bar{s}-1) \zeta(\bar{s}-1)}{\zeta(2 \bar{s}) \zeta(\bar{s}+1)} \operatorname{det}\left(\operatorname{Im}\left(\begin{array}{cc}
z & 0 \\
0 & w
\end{array}\right)\right)^{(\bar{s}-k+1) / 2} \operatorname{det}\left(2 \operatorname{Im}\left(\begin{array}{ll}
z & 0 \\
0 & w
\end{array}\right)\right)^{-\bar{s}+(1 / 2)}
\end{aligned}
$$

and

$$
\begin{aligned}
F_{k, v, \lambda}^{(2)} & \left(\left(\begin{array}{cc}
-\bar{z} & 0 \\
0 & w
\end{array}\right), \frac{\bar{s}-k+1}{2}\right) \\
\quad= & \left.\sum_{h \in \Lambda_{\lambda}^{*}} \sum_{r \in Z_{p r m m}^{(2, \lambda)} / G L_{\lambda}(Z)} b_{k, v, \lambda}^{2}\left(h{ }^{t} r\right], \operatorname{Im}\left(\begin{array}{cc}
z & 0 \\
0 & w
\end{array}\right), \frac{\bar{s}-k+1}{2}\right) \boldsymbol{e}\left(\sigma\left(h{ }^{t} r\right] \operatorname{Re}\left(\begin{array}{cc}
-\bar{z} & 0 \\
0 & w
\end{array}\right)\right)
\end{aligned}
$$

for $1 \leqq \lambda \leqq v \leqq 2$.

\section{Here}

$$
\begin{aligned}
\left.b_{k, 1,1}^{(2)}\left(h{ }^{t} r\right], \operatorname{Im}\left(\begin{array}{cc}
z & 0 \\
0 & w
\end{array}\right), \frac{\bar{s}-k+1}{2}\right) \\
=(-1)^{k / 2} 2 \pi \frac{1}{\Gamma((\bar{s}-k+1) / 2) \Gamma((\bar{s}+k+1) / 2)} S_{1}(h, \bar{s}+1) \\
\cdot \operatorname{det}\left(\operatorname{Im}\left(\begin{array}{cc}
z & 0 \\
0 & w
\end{array}\right)\right)^{(\bar{s}-k+1) / 2} \\
\cdot \eta_{1}\left(2 \operatorname{Im}\left(\begin{array}{cc}
z & 0 \\
0 & w
\end{array}\right)[r], \pi h, \frac{\bar{s}+k+1}{2}, \frac{\bar{s}-k+1}{2}\right)
\end{aligned}
$$




$$
\begin{aligned}
= & S_{1}(h, \bar{s}+1) \operatorname{det}\left(\operatorname{Im}\left(\begin{array}{cc}
z & 0 \\
0 & w
\end{array}\right)\right)^{(\bar{s}-k+1) / 2} \\
& \cdot \xi_{1}\left(\operatorname{Im}\left(\begin{array}{cc}
z & 0 \\
0 & w
\end{array}\right)[r], h, \frac{\bar{s}+k+1}{2}, \frac{\bar{s}-k+1}{2}\right),
\end{aligned}
$$

$$
\begin{aligned}
& \left.b_{k, 2,1}^{(2)}\left(h{ }^{t} r\right], \operatorname{Im}\left(\begin{array}{cc}
z & 0 \\
0 & w
\end{array}\right), \frac{\bar{s}-k+1}{2}\right) \\
& =(-1)^{k} 2^{2} \pi^{5 / 2} \frac{\Gamma(\bar{s}-(1 / 2)) \cdot S_{2}\left(\left(\begin{array}{ll}
h & 0 \\
0 & 0
\end{array}\right), \bar{s}+1\right)}{\Gamma((\bar{s}-k+1) / 2) \Gamma((\bar{s}-k) / 2) \Gamma((\bar{s}+k+1) / 2) \Gamma((\bar{s}+k) / 2)} \\
& \cdot \operatorname{det}\left(\operatorname{Im}\left(\begin{array}{cc}
z & 0 \\
0 & w
\end{array}\right)\right)^{(\bar{s}-k+1) / 2} \operatorname{det}\left(2 \operatorname{Im}\left(\begin{array}{cc}
z & 0 \\
0 & w
\end{array}\right)[r]\right)^{-1 / 2} \\
& \cdot \zeta_{1}^{(1)}\left(2 g\left(y, u_{r}\right), \bar{s}-\frac{1}{2}\right) \eta_{1}\left(2 \operatorname{Im}\left(\begin{array}{cc}
z & 0 \\
0 & w
\end{array}\right)[r], \pi h, \frac{\bar{s}+k}{2}, \frac{\bar{s}-k}{2}\right) \\
& =(-1)^{k / 2} 2 \pi^{3 / 2} \frac{\Gamma(\bar{s}-(1 / 2))}{\Gamma((\bar{s}-k+1) / 2) \Gamma((\bar{s}+k+1) / 2)} \cdot \frac{\zeta(2 \bar{s}-1) d_{1-\bar{s}}(h)}{\zeta(2 \bar{s}) \zeta(\bar{s}+1)} \\
& \cdot \operatorname{det}\left(\operatorname{Im}\left(\begin{array}{cc}
z & 0 \\
0 & w
\end{array}\right)\right)^{(\bar{s}-k+1) / 2} \operatorname{det}\left(2 \operatorname{Im}\left(\begin{array}{cc}
z & 0 \\
0 & w
\end{array}\right)\right)^{(1 / 2)-\bar{s}} \\
& \cdot \operatorname{det}\left(2 \operatorname{Im}\left(\begin{array}{cc}
z & 0 \\
0 & w
\end{array}\right)[r]\right)^{\bar{s}-1} \zeta_{1}\left(\operatorname{Im}\left(\begin{array}{cc}
z & 0 \\
0 & w
\end{array}\right)[r], h, \frac{\bar{s}+k}{2}, \frac{\bar{s}-k}{2}\right)
\end{aligned}
$$

and

$$
\begin{aligned}
b_{k, 2,2}^{(2)}\left(h\left[{ }^{t} r\right], \operatorname{Im}\left(\begin{array}{cc}
z & 0 \\
0 & w
\end{array}\right), \frac{\bar{s}-k+1}{2}\right) \\
=(-1)^{k} 2^{2} \pi^{2} \frac{1}{\Gamma((\bar{s}-k+1) / 2) \Gamma((\bar{s}-k) / 2) \Gamma((\bar{s}+k+1) / 2) \Gamma((\bar{s}+k) / 2)} \\
\cdot S_{2}(h, \bar{s}+1) \operatorname{det}\left(\operatorname{Im}\left(\begin{array}{cc}
z & 0 \\
0 & w
\end{array}\right)\right)^{(\bar{s}-k+1) / 2} \\
\cdot \eta_{2}\left(2 \operatorname{Im}\left(\begin{array}{cc}
z & 0 \\
0 & w
\end{array}\right)[r], \pi h, \frac{\bar{s}+k+1}{2}, \frac{\bar{s}-k+1}{2}\right)
\end{aligned}
$$




$$
\begin{aligned}
= & S_{2}(h, \bar{s}+1) \operatorname{det}\left(\operatorname{Im}\left(\begin{array}{cc}
z & 0 \\
0 & w
\end{array}\right)\right)^{(\bar{s}-k+1) / 2} \\
& \cdot \xi_{2}\left(\operatorname{Im}\left(\begin{array}{ll}
z & 0 \\
0 & w
\end{array}\right)[r], h, \frac{\bar{s}+k+1}{2}, \frac{\bar{s}-k+1}{2}\right) .
\end{aligned}
$$

In the following, we denote $\mu(h g)$ (resp. $\lambda(h g)$ ) the smallest (largest) eigenvalue of nonzero eigenvalues of $h\left[g^{1 / 2}\right]$ for $0 \neq h \in V_{m}$ and $g \in P_{m}$.

Proposition 1. Let $s \in C$ and assume $\zeta(2 s) \neq 0$. Then for positive matrix $\left(\begin{array}{ll}t & 0 \\ 0 & y\end{array}\right)$, there exists a constant $c \in \boldsymbol{R}_{>0}$ depending only on $\operatorname{Re}(s)=\sigma$ such that

$$
\left|\zeta_{1}^{(2)}\left(\left(\begin{array}{ll}
t & 0 \\
0 & y
\end{array}\right), s\right)\right| \leqq c\left(y^{-2 \sigma-1}+y^{-2 \sigma+1}+y^{-2}+t^{-2 \sigma-1}+t^{-2 \sigma+1}+t^{-2}\right) .
$$

Proof. By [Ma, p. 284], for $g \in P_{2}$

$$
\Xi_{1}^{(2)}(g, s):=2 \pi^{-s} s(1-s) \Gamma(s) \zeta(2 s) \zeta_{1}^{(2)}(g, s)
$$

has an integral representation

$$
\Xi_{1}^{(2)}(g, s)=\int_{\operatorname{det}(y) \geqq 0}^{R_{1}}\left\{\Theta(y, g) \operatorname{det}(y)^{s}+\operatorname{det}(g)^{-1 / 2} \Theta\left(y, g^{-1}\right) \operatorname{det}(y)^{1-s}\right\} \operatorname{det}(y)^{-1} d y .
$$

Here $R_{1}$ is the Minkowski reduced domain in $P_{1}$ and

$$
\Theta\left(y^{(1)}, g^{(2)}\right):=\sum_{\substack{c \in Z^{(2,1)} \\ \text { rank } c=1}} \boldsymbol{P}_{y} e^{-\pi \sigma(g[c] y)}
$$

with a certain differential operator $\boldsymbol{P}_{y}$ of degree 2. The expression (4.6) shows that $\Xi_{1}^{(2)}(g, s)$ is an entire function of $s \in C$.

By [Mi, Proposition 4.4] and [K1, p. 145], there exist constants $c_{1}, c_{2}$ depending only on a positive constant $r$ with $\left|\operatorname{det}\left(\operatorname{Re}(z)^{-1} z\right)\right| \leqq r$ such that

$$
\begin{aligned}
\Xi_{1}^{(2)}(g, s) \leqq & c_{1} \int_{1}^{\infty}\left\{\mu(g)^{-1} t^{\sigma-2} \exp \left(-c_{2} \mu(g) t\right)\right. \\
& \left.+\operatorname{det}(g)^{-1 / 2} \lambda(g) t^{-\sigma-1} \exp \left(-c_{2} \lambda(g)^{-1} t\right)\right\} d t
\end{aligned}
$$

It is easy to see that there exist positive constants $c_{3}$ depending only on $\sigma$ such that

$$
\int_{1}^{\infty} t^{\sigma} \exp \left(-\alpha t^{1 / v}\right) d t \leqq c_{3}\left\{\alpha^{-v(\sigma+1)-1}+\alpha^{-v(\sigma+1)+1}+\alpha^{-1}+\alpha\right\}
$$

for all $\alpha \in \boldsymbol{R}_{>0}$. The Proposition follows by using the last inequality. 
Proposition 2. For $y>0, h \in Z_{\neq 0}$ and $(\alpha, \beta) \in C^{2}, \xi_{1}(y, h, \alpha, \beta)$ is a holomorphic function of $(\alpha, \beta)$ on $C^{2}$. Let $T$ be a compact subset of $C^{2}$. Then there exist positive constants $c_{1}$ and $c_{2}$ depending only on $T$ such that

$$
\left|\xi_{1}(y, h, \alpha, \beta)\right| \leqq \begin{cases}c_{1} e^{-2 \pi h y} h^{\operatorname{Re}(\alpha)-1} y^{-\operatorname{Re}(\beta)}\left(1+y^{-c_{2}}\right) & \text { for } h>0 \\ c_{1} e^{-2 \pi|h| y}|h|^{\operatorname{Re}(\beta)-1} y^{-\operatorname{Re}(\alpha)}\left(1+y^{-c_{2}}\right) & \text { for } h<0\end{cases}
$$

with $(\alpha, \beta) \in T$. We are able to take

$$
c_{2}=\max \{[\operatorname{Re}(\alpha)],-\min \{0,[\operatorname{Re}(\beta)]\}\}
$$

where $[\operatorname{Re}(\alpha)]$ denotes the largest integer l such that $l \leqq \operatorname{Re}(\alpha)$.

Proof. Above results are well hnown. For example, see [Miy, p. 281].

Proposition 3. For $y \in P_{2}, h \in \Lambda_{2}^{*}$ and $(\alpha, \beta) \in C^{2} \backslash \boldsymbol{R}^{2}, \xi_{2}(y, h, \alpha, \beta)$ is a holomorphic function of $(\alpha, \beta)$ on $\boldsymbol{C}^{2} \backslash \boldsymbol{R}^{2}$. Let $r$ be a positive constant and $T$ a compact subset of $\boldsymbol{C}^{2}$. Then there exist positive constants $c_{1}, c_{2}$ and $c_{3}$ depending only on $r$ and $T$ such that

$$
\left|\xi_{2}(y, h, \alpha, \beta)\right| \leqq c_{1} e^{-\tau(2 \pi h y)} \sigma(y)^{c_{2}}\left(\lambda(h y)^{c_{3}}+\mu(h y)^{-c_{3}}\right)
$$

for $\mu(y) \geqq r$ and $(\alpha, \beta) \in T$. Here $\tau(h y)$ is the sum of absolute values of eigenvalues of hy.

Proof. The fundamental properties of the confluent hypergeometric functions $\xi_{m}$ or $\eta_{m}$ are due to [Sh 2].

THEOREM 2. For $s \in C$ in $0<\operatorname{Re}(s)<1, E_{k}^{(2)}\left(\left(\begin{array}{cc}-\bar{z} & 0 \\ 0 & w\end{array}\right),(\bar{s}-k+1) / 2\right)$ is a $C^{\infty}$ automorphic form of bounded growth except on the pole.

Proof. By Theorem B (2), $E_{k}^{(2)}\left(\left(\begin{array}{cc}z & 0 \\ 0 & w\end{array}\right), s\right)$ is a $C^{\infty}$-modular form of onevariable both $z$ and $w$ on $S L(2, Z)$. We shall show the growth condition of $E_{k}^{(2)}\left(\left(\begin{array}{cc}z & 0 \\ 0 & w\end{array}\right), s\right)$.

Let $\operatorname{Im}(z)=t$ and $\operatorname{Im}(w)=y . \quad$ By (4.0.0), (4.1.0), (4.2.0) and Proposition 1, there exist positive constants $c_{1}, \ldots, c_{4}$ depending only on $\operatorname{Im}(z)=t, \operatorname{Re}(s)=\sigma$ and weight $k$ such that

$$
\begin{aligned}
& \left|F_{k, 0,0}^{(2)}\left(\left(\begin{array}{cc}
-\bar{z} & 0 \\
0 & w
\end{array}\right), \frac{\bar{s}-k+1}{2}\right)\right| \leqq c_{1} \cdot y^{(\sigma-k+1) / 2}, \\
& \left|F_{k, 1,0}^{(2)}\left(\left(\begin{array}{cc}
-\bar{z} & 0 \\
0 & w
\end{array}\right), \frac{\bar{s}-k+1}{2}\right)\right| \leqq c_{2} \cdot y^{(\sigma-k+1) / 2}\left(y^{-2 \sigma-1}+y^{-2 \sigma+1}+y^{-2}+c_{3}\right),
\end{aligned}
$$




$$
\left|F_{k, 2,0}^{(2)}\left(\left(\begin{array}{cc}
-\bar{z} & 0 \\
0 & w
\end{array}\right), \frac{\bar{s}-k+1}{2}\right)\right| \leqq c_{4} \cdot y^{(-\sigma-k+2) / 2} .
$$

Here we assumed $\zeta(2 s) \zeta(s+1) \neq 0$ and $s \in \boldsymbol{C} \backslash \boldsymbol{R}$. By (4.1.1), (4.2.1) and Proposition 2 , there exist positive constants $c_{5}, \ldots, c_{10}$ depending only on $h, \operatorname{Im}(z)=$ $t, \operatorname{Re}(s)=\sigma$ and weight $k$, and exist constants $\left(r_{1}, r_{2}, r_{3}, r_{4}\right) \in \boldsymbol{R}^{4}$ depending only on $\sigma$ and weight $k$ such that

$$
\begin{aligned}
& \left|F_{k, 1,1}^{(2)}\left(\left(\begin{array}{cc}
-\bar{z} & 0 \\
0 & w
\end{array}\right), \frac{\bar{s}-k+1}{2}\right)\right| \\
& \quad \leqq \sum_{\substack{h \in \boldsymbol{Z}_{\neq 0} \\
r \in \boldsymbol{Z}_{\text {prm }}^{(2,1)} / \pm 1}} c_{5} \cdot y^{r_{1}}|h|^{c_{6}}\left(\begin{array}{ll}
t & 0 \\
0 & y
\end{array}\right)[r]^{r_{2}}\left(1+\left(\begin{array}{ll}
t & 0 \\
0 & y
\end{array}\right)[r]^{-c_{7}}\right) e^{-2 \pi|h|\left(\begin{array}{ll}
1 & 0 \\
0 & y
\end{array}\right)[r]}, \\
& \left.\quad \leqq \sum_{\substack{h \in \boldsymbol{Z}_{\neq 0} \\
r \in \boldsymbol{Z}_{p r m}^{(2,1)} / \pm 1}}^{(2)} c_{8,2} \cdot\left(\begin{array}{cc}
-\bar{z} & 0 \\
0 & w
\end{array}\right), \frac{\bar{s}-k+1}{2}\right) \mid
\end{aligned}
$$

Thus $\left|F_{k, 1,1}^{(2)}\right|$ and $\left|F_{k, 2,1}^{(2)}\right| \rightarrow 0$ when $y \rightarrow \infty . \quad$ By (4.2.2) and Proposition 3, there exist positive constants $c_{11}, c_{12}$ and $c_{13}$ depending only on $h, \operatorname{Im}(z)=t, \operatorname{Re}(s)=\sigma$, $\rho>0$ with $\mu\left(\left(\begin{array}{ll}t & 0 \\ 0 & y\end{array}\right)\right)>\rho$ and weight $k$; and exist constant $r_{5} \in \boldsymbol{R}$ depending only on $\rho$ and weight $k$ such that

$$
\begin{aligned}
\left|F_{k, 2,2}^{(2)}\left(\left(\begin{array}{cc}
-\bar{z} & 0 \\
0 & w
\end{array}\right), \frac{\bar{s}-k+1}{2}\right)\right| \leqq & \sum_{h \in \Lambda_{2}^{*}} c_{11} \cdot y^{r_{5}} e^{-\tau\left(2 \pi h\left(\begin{array}{l}
t \\
0
\end{array}\right)\right)} \sigma\left(\left(\begin{array}{ll}
t & 0 \\
0 & y
\end{array}\right)\right)^{c_{12}} \\
& \times\left(\lambda\left(h\left(\begin{array}{ll}
t & 0 \\
0 & y
\end{array}\right)\right)^{c_{13}}+\mu\left(h\left(\begin{array}{ll}
t & 0 \\
0 & y
\end{array}\right)\right)^{-c_{13}}\right) .
\end{aligned}
$$

Let $g \in P_{m}$. By [Sh 2, Lemma 2.9], there exists an element $b \in G L_{m}(Z)$ such that

$$
g\left[{ }^{t} b\right]=\operatorname{diag}\left(g_{1}, \ldots, g_{m}\right), \quad g_{j} \in \boldsymbol{R}_{>0}
$$

and

$$
h\left[b^{-1}\right]=\operatorname{diag}\left(1_{p},-1_{q}\right)
$$

with some $p, q \in Z_{\geqq 0}, p+q=m$. Hence 


$$
\begin{aligned}
\tau(h g) & =\tau\left(\operatorname{diag}\left(1_{p},-1_{q}\right) \cdot \operatorname{diag}\left(g_{1}, \ldots, g_{m}\right)\right) \\
& =\sum_{J=1}^{m} g_{j} \\
& \geqq m \prod_{j=1}^{m} g_{j}^{1 / m} \\
& =m|\operatorname{det}(h)|^{1 / m} \operatorname{det}(g)^{1 / m} .
\end{aligned}
$$

Therefore in our case

$$
\tau\left(h\left(\begin{array}{cc}
t & 0 \\
0 & y
\end{array}\right)\right) \geqq 2|\operatorname{det}(h)|^{1 / 2}(t y)^{1 / 2}
$$

hence $\left|F_{k, 2,2}^{(2)}\right| \rightarrow 0$ when $y \rightarrow \infty$.

Now we estimate the Eisenstein series using we have obtained. There exist positive constants $a_{1}, \ldots, a_{4}$ depending only on $\operatorname{Im}(z)=t, \operatorname{Re}(s)=\sigma$ and weight $k$ such that

$$
\begin{aligned}
\left|E_{k}^{(2)}\left(\left(\begin{array}{cc}
-\bar{z} & 0 \\
0 & w
\end{array}\right), \frac{\bar{s}-k+1}{2}\right)\right|= & \left|\sum_{v=0}^{2} \sum_{\lambda=0}^{v} F_{k, v, \lambda}^{(2)}\left(\left(\begin{array}{cc}
-\bar{z} & 0 \\
0 & w
\end{array}\right), \frac{\bar{s}-k+1}{2}\right)\right| \\
\leqq & a_{1} \cdot y^{(\sigma-k+1) / 2}\left(y^{-2 \sigma-1}+y^{-2 \sigma+1}+y^{-2}+a_{2}\right) \\
& +a_{3} \cdot y^{(-\sigma-k+2) / 2}
\end{aligned}
$$

and especially in the region $0<\operatorname{Re}(s)<1$ and for $y \rightarrow \infty$

$$
\leqq a_{4} \cdot y^{(-k+3) / 2}
$$

Next, we study in the case $y \rightarrow 0$ following the proof of [St, Proposition 2]. Let $\varphi(w)=y^{k / 2} E_{k}^{(2)}\left(\left(\begin{array}{cc}-\bar{z} & 0 \\ 0 & w\end{array}\right),(\bar{s}-k+1) / 2\right)$ with $y=\operatorname{Im}(w)$. Then

$$
|\varphi(\gamma(w))|=|\varphi(w)|
$$

for all $\gamma \in \Gamma^{1}=S L(2, Z)$ and $w \in H_{1}$. We choose $\Gamma^{1} \backslash H_{1}$ such that $|w| \geqq 1$ and $|\operatorname{Re}(w)| \leqq 1$ for $w \in \Gamma^{1} \backslash H_{1}$. By (4.7)

$$
|\varphi(w)|=\left|y^{k / 2} E_{k}^{(2)}\left(\left(\begin{array}{cc}
-\bar{z} & 0 \\
0 & w
\end{array}\right), \frac{\bar{s}-k+1}{2}\right)\right| \leqq a_{4} \cdot y^{((-k+3) / 2)+(k / 2)} .
$$

Let $w=x+i y \in H_{1}$ and choose $g=\left(\begin{array}{ll}a & b \\ c & d\end{array}\right) \in \Gamma^{1}$ such that $g(w) \in$ $\Gamma^{1} \backslash H_{1}$. Then 


$$
\begin{aligned}
|\varphi(w)| & =|\varphi(g(w))| \\
& \leqq a_{4} \cdot|\operatorname{Im}(g(w))|^{3 / 2} \\
& =a_{4} \cdot y^{3 / 2}|c z+d|^{-3} \\
& \leqq a_{4} \cdot\left(y^{-3 / 2}+y^{3 / 2}\right)
\end{aligned}
$$

Therefore we have

$$
\left|E_{k}^{(2)}\left(\left(\begin{array}{cc}
-\bar{z} & 0 \\
0 & w
\end{array}\right), \frac{\bar{s}-k+1}{2}\right)\right| \leqq a_{4} \cdot\left(y^{(-k-3) / 2}+y^{(-k+3) / 2}\right)
$$

for any $y>0$ and $0<\operatorname{Re}(s)<1$. This completes the proof of Theorem 2 .

\section{Proof of Theorem 1}

First, we show the following Lemma.

Lemma 1. (1) Put $(\alpha, \beta) \in C^{2} \backslash \boldsymbol{R}^{2}$ with $k+\beta-\alpha=0$. Then for any $n \in$ $\boldsymbol{Z}_{>0}$,

$$
\int_{0}^{\infty} e^{-2 \pi n y} y^{k-2+\beta} \xi_{1}(y, n, \alpha, \beta) d y=0
$$

(2) Put $(\theta, \gamma, \alpha, \beta) \in C^{4}$ with $\theta-\alpha-\beta+1=0$ and $k-1+\beta+\gamma=0$. For given $n \in Z_{>0}$, put $(h, p, q) \in Z_{>0}^{3}$ such that $h q^{2}=n$. Then we have for any $t>0$,

$$
\int_{0}^{\infty} e^{-2 \pi n y} y^{k-2+\gamma}\left(p^{2} t+q^{2} y\right)^{\theta} \xi_{1}\left(p^{2} t+q^{2} y, h, \alpha, \beta\right) d y=0 .
$$

(3) Put $(\alpha, \beta) \in C^{2}$ with $k+\beta-\alpha=0$. For given $n \in Z_{>0}$, put $(h, p, q) \in$ $Z_{>0}^{3}$ such that $h q^{2}=n$. Then we have for any $t>0$,

$$
\begin{aligned}
& \int_{0}^{\infty} e^{-2 \pi n y} y^{k-2+\beta} \xi_{1}\left(p^{2} t+q^{2} y, h, \alpha, \beta\right) d y \\
& \quad=e^{(\pi / 2) i(\beta-\alpha)} 2^{-\alpha-\beta+1} \pi p^{-2 \beta} q^{-2 \alpha+2} \Gamma(\alpha-1) \Gamma(\alpha)^{-1} t^{-\beta} e^{-2 \pi h p^{2} t}
\end{aligned}
$$

Proof. (1) By (2.3),

$$
\begin{aligned}
& \int_{0}^{\infty} e^{-2 \pi n y} y^{k-2+\beta} \xi_{1}(y, n, \alpha, \beta) d y \\
& \quad=\int_{-\infty}^{\infty} \int_{0}^{\infty}(x+i)^{-\alpha}(x-i)^{-\beta} e^{-2 \pi n(1+c x) y} y^{k-1-\alpha} d y d x
\end{aligned}
$$




$$
\begin{aligned}
& =(2 \pi i n)^{-k-\gamma+\alpha+\beta} \Gamma(k-\alpha) \int_{-\infty}^{\infty}(x+i)^{-\alpha}(x-i)^{-k+\alpha} d x \\
& =2^{2-k-\beta} \pi i^{-\alpha \beta}(2 \pi n)^{-k+\alpha} \Gamma(k-\alpha) \Gamma(k+\beta-1) \Gamma(k+\beta-\alpha)^{-1} \Gamma(\alpha)^{-1} .
\end{aligned}
$$

Thus (5.1) is hold for $k+\beta-\alpha=0$.

(2) For $\theta-\alpha-\beta=1$,

$$
\begin{aligned}
& \int_{0}^{\infty} e^{-2 \pi n y} y^{k-2+\gamma}\left(p^{2} t+q^{2} y\right)^{\theta} \xi_{1}\left(p^{2} t+q^{2} y, h, \alpha, \beta\right) d y \\
& \quad=\int_{-\infty}^{\infty} \int_{0}^{\infty}(x+i)^{-\alpha}(x-i)^{-\beta}\left(p^{2} t+q^{2} y\right)^{\theta+1-\alpha-\beta} e^{-2 \pi n y-2 \pi i h\left(p^{2} t+q^{2} y\right) x} y^{k-2+\gamma} d y d x \\
& \quad=(2 \pi i n)^{-k+1-\gamma} \Gamma(k-1+\gamma) \int_{-\infty}^{\infty}(x+i)^{-\alpha} e^{-2 \pi i h p^{2} t x} d x .
\end{aligned}
$$

Here we use the relation:

$$
\int_{-\infty}^{\infty} e^{i u v}(a+i v)^{-\mu} d v= \begin{cases}2 \pi u^{\mu-1} e^{-a u} \Gamma(\mu)^{-1} & \text { for } u>0 \\ 0 & \text { for } u<0\end{cases}
$$

(3) By (2.4),

$$
\begin{aligned}
& \int_{0}^{\infty} e^{-2 \pi n y} y^{k-2+\beta} \xi_{1}\left(p^{2} t+q^{2} y, h, \alpha, \beta\right) d y \\
& \quad=e^{(\pi / 2) i(\beta-\alpha)} 2 \pi \Gamma(\alpha)^{-1} \Gamma(\beta)^{-1} \int_{0}^{\infty} e^{-2 \pi n y} y^{k-2+\beta} \eta_{1}\left(p^{2} t+q^{2} y, \pi h, \alpha, \beta\right) d y
\end{aligned}
$$

For $k+\beta-\alpha=0$,

$$
\begin{aligned}
& \int_{0}^{\infty} e^{-2 \pi n y} y^{k-2+\beta} \eta_{1}\left(p^{2} t+q^{2} y, \pi h, \alpha, \beta\right) d y \\
& \quad=\int_{x \pm \pi h>0} \int_{0}^{\infty}(x+\pi h)^{\alpha-1}(x-\pi h)^{\beta-1} e^{-2 \pi n y-2\left(p^{2} t+q^{2} y\right) x} y^{k-2+\beta} d y d x \\
& \quad=\left(2 q^{2}\right)^{-\alpha+1} \Gamma(\alpha-1) \int_{x-\pi h>0}(x-\pi h)^{\beta-1} e^{-2 t p^{2} x} d x \\
& \quad=\left(2 q^{2}\right)^{-\alpha+1}\left(2 p^{2}\right)^{-\beta} t^{-\beta} \Gamma(\alpha-1) \Gamma(\beta) e^{-2 \pi t h p^{2}}
\end{aligned}
$$

Thus we obtain the assertion of Lemma 1.

Proof of Theorem 1. First we write the Fourier expansion of $E_{k}^{(2)}\left(\left(\begin{array}{cc}-\bar{z} & 0 \\ 0 & w\end{array}\right),(\bar{s}-k+1) / 2\right)$ respect to $w=x+i y$ as follows: 


$$
E_{k}^{(2)}\left(\left(\begin{array}{cc}
-\bar{z} & 0 \\
0 & w
\end{array}\right), \frac{\bar{s}-k+1}{2}\right)=\sum_{n=-\infty}^{\infty} a(n, s, \operatorname{Im}(w)) e^{2 \pi i n \operatorname{Re}(w)}
$$

By Theorem B (2), $E_{k}^{(2)}\left(\left(\begin{array}{cc}-\bar{z} & 0 \\ 0 & w\end{array}\right),(\bar{s}-k+1) / 2\right)$ is a $C^{\infty}$ automorphic form of weight $k$ in each of the variables $z$ and $w$ for any $s \in C$ except the poles of $E_{k}^{(2)}\left(\left(\begin{array}{cc}-\bar{z} & 0 \\ 0 & w\end{array}\right),(\bar{s}-k+1) / 2\right)$. And by Theorem $2 E_{k}^{(2)}\left(\left(\begin{array}{cc}-\bar{z} & 0 \\ 0 & w\end{array}\right),(\bar{s}-k+1) / 2\right)$ is of bounded growth for $0<\operatorname{Re}(s)<1$ except on the pole. The constant terms of the Fourier expansion of $E_{k}^{(2)}$ show that the possible poles arise when $\zeta(2 s)=0$ in the region $0<\operatorname{Re}(s)<1$ with $s \notin \boldsymbol{R}$. By Theorem A, there exists $h(z, s)=\sum_{n=1}^{\infty} c(n, s) e^{2 \pi i n z} \in S_{k}^{1} \quad$ such that $\left\langle g, E_{k}^{(2)}\left(\left(\begin{array}{cc}-\bar{z} & 0 \\ 0 & w\end{array}\right),(\bar{s}-k+1) / 2\right)\right\rangle=$ $\langle g, h\rangle$ for all $g \in S_{k}^{1}$.

Here

$$
c(n, s):=(4 \pi n)^{k-1} \Gamma(k-1)^{-1} \int_{0}^{\infty} a(n, s, y) e^{-2 \pi n y} \operatorname{det}(y)^{k-2} d y .
$$

By (4.4)

$$
\begin{aligned}
a(n, s, \operatorname{Im}(w))= & \sum_{v=1}^{2} \sum_{\lambda=1}^{v} \sum_{\left.\begin{array}{c}
h \in \Lambda_{\lambda}^{*} \\
\left.h{ }^{t} r\right]=\left(\begin{array}{c}
* \\
*
\end{array}\right.
\end{array}\right)} \sum_{r \in \boldsymbol{Z}_{p r m m}^{(2, \lambda)} / G L_{\lambda}(\boldsymbol{Z})} \\
& \left.\times b_{k, v, \lambda}^{(2)}\left(h{ }^{t} r\right], \operatorname{Im}\left(\begin{array}{cc}
z & 0 \\
0 & w
\end{array}\right), \frac{\bar{s}-k+1}{2}\right) \boldsymbol{e}\left(\sigma\left(h\left[{ }^{t} r\right] \operatorname{Re}\left(\begin{array}{cc}
-\bar{z} & 0 \\
0 & 0
\end{array}\right)\right)\right)
\end{aligned}
$$

for $n \neq 0$. In the following we put $\operatorname{Re}(z)=0$ and $\operatorname{Im}(z)=t$. Using (4.1.1)(4.2.2) and Lemma 1, we have

$$
\begin{aligned}
c(n, s) \times(4 \pi n)^{1-k} \Gamma(k-1) & \sum_{v=1}^{2} \sum_{\lambda=1}^{v} \int_{0}^{\infty} \sum_{\substack{\left.h \in \Lambda_{\lambda}^{*} \\
h{ }^{t} r\right]=\left(\begin{array}{c}
* \\
* \\
*
\end{array}\right)}} \sum_{r \in Z_{p r m}^{(2, \lambda)} / G L_{\lambda}(Z)} \\
& \times b_{k, v, \lambda}^{(2)}\left(h\left[{ }^{t} r\right], \operatorname{Im}\left(\begin{array}{cc}
z & 0 \\
0 & w
\end{array}\right), \frac{\bar{s}-k+1}{2}\right) e^{-2 \pi n y} y^{k-2} d y
\end{aligned}
$$




$$
\begin{aligned}
& =\sum_{\substack{\left.h \in \Lambda_{1}^{*} \\
h{ }^{t} r\right]=\left(\begin{array}{c}
* \\
* \\
*
\end{array}\right)}} \sum_{r \in \boldsymbol{Z}_{p r m}^{(2,1)} / G L_{1}(\boldsymbol{Z})} S_{1}(h, \bar{s}+1) t^{(\bar{s}-k+1) / 2} \\
& \times \int_{0}^{\infty} e^{-2 \pi n y} y^{k-2+(s-k+1) / 2} \xi_{1}\left(\left(\begin{array}{ll}
t & 0 \\
0 & y
\end{array}\right)[r], h, \frac{\bar{s}+k+1}{2}, \frac{\bar{s}-k+1}{2}\right) d y \\
& +\sum_{\substack{\left.h \in \Lambda_{1}^{*} \\
h{ }^{t} r\right]=\left(\begin{array}{c}
* \\
* \\
*
\end{array}\right)}} \sum_{r \in \boldsymbol{Z}_{p r m}^{(2,1)} / G L_{1}(\boldsymbol{Z})}(-1)^{k / 2} 2^{2-2 \bar{s}} \pi^{3 / 2} \\
& \times \frac{\Gamma(\bar{s}-(1 / 2))}{\Gamma((\bar{s}-k+1) / 2) \Gamma((\bar{s}+k+1) / 2)} S_{2}\left(\left(\begin{array}{ll}
h & 0 \\
0 & 0
\end{array}\right), \bar{s}+1\right) \\
& \times \int_{0}^{\infty} e^{-2 \pi n y} y^{k-2} \operatorname{det}\left(\left(\begin{array}{ll}
t & 0 \\
0 & y
\end{array}\right)\right)^{(-\bar{s}-k+2) / 2} \operatorname{det}\left(2\left(\begin{array}{ll}
t & 0 \\
0 & y
\end{array}\right)[r]\right)^{\bar{s}-1} \\
& \times \xi_{1}\left(\left(\begin{array}{ll}
t & 0 \\
0 & y
\end{array}\right)[r], h, \frac{\bar{s}+k}{2}, \frac{\bar{s}-k}{2}\right) d y \\
& +\int_{0}^{\infty} \sum_{\substack{h \in \Lambda_{2}^{*} \\
h\left[{ }^{t} r\right]=\left(\begin{array}{c}
* \\
* \\
*
\end{array}\right)}} \sum_{r \in Z_{p r m}^{(2,2)} / G L_{2}(\boldsymbol{Z})} S_{2}(h, \bar{s}+1) t^{(\bar{s}-k+1) / 2} \\
& \times e^{-2 \pi n y} y^{k-2+(\bar{s}-k+1) / 2} \xi_{2}\left(\left(\begin{array}{ll}
t & 0 \\
0 & y
\end{array}\right)[r], h, \frac{\bar{s}+k+1}{2}, \frac{\bar{s}-k+1}{2}\right) d y \\
& =\sum_{\substack{h, p, q \in \boldsymbol{Z}_{>0} \\
h q^{2}=n(p, q)=1}}(-1)^{k / 2} 2^{-\bar{s}} \pi \cdot \frac{\Gamma((\bar{s}+k-1) / 2)}{\Gamma((\bar{s}+k+1) / 2)} \frac{d_{-\bar{s}}(h)}{\zeta(\bar{s}+1)} \\
& \times t^{(\bar{s}-k+1) / 2} p^{-\bar{s}+k-1} q^{-\bar{s}-k+1} e^{-2 \pi t h p^{2}} \\
& +\int_{0}^{\infty} \sum_{h \in \Lambda_{2}^{*} h=\left(\begin{array}{c}
* \\
* \\
n
\end{array}\right)} t^{(\bar{s}-k+1) / 2} S_{2}(h, \bar{s}+1) y^{(\bar{s}+k-3) / 2} e^{-2 \pi n y} \\
& \times \xi_{2}\left(\left(\begin{array}{ll}
t & 0 \\
0 & y
\end{array}\right), h, \frac{\bar{s}+k+1}{2}, \frac{\bar{s}-k+1}{2}\right) d y .
\end{aligned}
$$

Here the interchange of summation and integration is justified by using Proposition 2. On the other hand, by (3.1) 


$$
\begin{aligned}
L(s, f, \underline{s}) \cdot f(z)= & (-1)^{k / 2} 2^{2 s+k-4} \pi^{-3 / 2} \Gamma\left(\frac{s+1}{2}\right) \Gamma\left(\frac{s+k}{2}\right) \Gamma\left(\frac{s+k+1}{2}\right) \\
& \times \Gamma(s+k-1)^{-1} \zeta(s+1)\left\langle f, E_{k}^{(2)}\left(\left(\begin{array}{cc}
-\bar{z} & 0 \\
0 & *
\end{array}\right), \frac{\bar{s}-k+1}{2}\right)\right\rangle .
\end{aligned}
$$

Therefore for $0<\operatorname{Re}(s)<1,\left\langle f, E_{k}^{(2)}\left(\left(\begin{array}{cc}-\bar{z} & 0 \\ 0 & *\end{array}\right),(\bar{s}-k+1) / 2\right)\right\rangle=\langle f, h\rangle=0$ if and only if $L(s, f, \underline{s t})=0$. From $\operatorname{dim} S_{k}^{1}=1$, we have $h(z, \rho)=0$, hence $c(n, \rho)=0$ for every positive integer $n$. Thus we conclude the proof of Theorem 1.

Acknowledgment. The author would like to thank Professor S. Mizumoto for his helpful advice.

\title{
REFERENCES
}

[An] A. N. Andrianov, The multiplicatıve arithmetic of Siegel modular forms, Russian Math. Surveys, 34 (1979), 75-148.

[Bö 1] S. BöCHERER, Über die Fourıerkoeffizıenten der Siegelschen Eisensteınreihen, Manuscrıpta Math., 45 (1984), 273-288.

[Bö 2] S. BÖChERER, ÜBER DIE FunkTIONALGLEICHUNG AUTOMORPHER L-Funktionen zur Siegelschen Modulgruppe, J. Reine Angew. Math., 362 (1985), 146-168.

[Ki] Y. KITAOKA, Dirıchlet series in the theory of Siegel modular forms, Nagoya Math. J., 95 (1984), 73-84.

[K1] H. KLINGEN, Introductory Lectures on Siegel Modular Forms, Cambridge Stud. Adv. Math., 20, Cambridge Unıv. Press, 1990.

[Ma] H. MAAss, Siegel's Modular Forms and Dirıchlet Serıes, Lecture Notes in Math., 216, Springer, 1971.

[Miy] T. MiYaKe, Modular Forms, Springer, 1989.

[Mi] S. Mızumoto, Eisenstein series for Siegel modular groups, Math. Ann., 297 (1993), 581625.

[Nd] T. NoDA, An application of the projections of $C^{\infty}$ automorphic forms, Acta Arith., 72 (1995), 229-234.

[Sh 1] G. ShimURA, On the holomorphy of certain Dirıchlet series, Proc. London Math. Soc., 31 (1975), 79-98.

[Sh 2] G. Shimura, Confluent hypergeometric functions on tube domains, Math. Ann., 260 (1982), 269-302.

[St] J. Sturm, The critical values of zeta functions associated to the symplectic group, Duke Math. J., 48 (1981), 327-350.

\author{
Department of Mathematics \\ FACULTY OF SCIENCE \\ Tokyo Institute of Technology \\ OH-OKayama, Meguro-KU, TOKYo, 152-8551 \\ JAPAN
}

